\title{
The ergonomic process of an automotive company in Brazil: a study case ${ }^{1}$
}

\author{
Carolina Bustos ${ }^{a *}$, Daniela Fischer ${ }^{a}$, Lucimara Ballardin $^{a}$ and Rudolf Nielsen ${ }^{a}$ \\ ${ }^{a}$ DFE Ergonomia de Produtos, Processos e Ambientes Ltda.
}

\begin{abstract}
The goal of this paper is to present the ergonomic process of an automotive company, whose focus is on the adaptation of the work conditions to the psychophysiological characteristics of its employees. The planning and the development of the ergonomic actions took place in three distinctive stages: ergonomic analysis of the work post (stage 1), ergonomic adaptations (stage 2) and Ergonomic Committee (stage 3). The activities started in June 2006 and have lasted to the current date, keeping a permanent improving process. The procedure adopted was based on the ergonomic analysis methodology proposed by Wisner (1994:1997) and the stages of the Ergonomic Analysis of Work presented in the 17 NR Regulatory Manual (MET, 2002). The paper's approach focused on the voluntary participation of workers from different areas and different hierarchical levels of the organization throughout all the stages of the process. The methodological procedures included descriptive research techniques, exploratory and qualitative research criteria, background and guidelines available in literature and legislation, as well as company information. Among the main results it can be mentioned the satisfaction of the employees regarding the appropriate work conditions, cultural and organizational changes and the creation of an Ergonomic Committee in the company.
\end{abstract}

Keywords: Ergonomic Analysis of Work, Ergonomic Process, Ergonomic Committee, Automotive Industry, Ergonomic Adaptations.

\section{Introduction}

The ergonomic process of the company focused on the application of the concept according to which ergonomics does not only derives from the knowledge developed by specialists in the field but from what workers have to say about it as well. After all, they are the ones who feel the problems originated from bad postures, repetitive operations, weight handling, among many others, occasionally intensified by the environment or any other factor.

The definition of Ergonomics itself, presented by Allain Wisner in 1987 - according to whom "Ergonomics is the setting of the whole of scientific knowledge relating to the Man and necessary to design tools, machines and devices which can be used by the greatest number with the maximum of comfort, safety and effectiveness" (MTE, 2002) - was simply and profoundly changed by the author in 1994, when he added, as relevant to the definition, the knowledge of the workers to the knowledge of the specialists. Thus "Ergonomics is the art in which are both used the techno scientific knowledge and the workers' knowledge about their own work situation" (MTE, 2002).

Therefore, the NR 17 Regulatory Manual, from the Ministry of Labor and Employment, which attends to Ergonomics, uses this last definition when puts both conceptions together as indispensable for the success of the ergonomic action.

Regarding the previous context, this paper objectives to present the ergonomic process of an automotive company, which is concerned about the adaptation of the work conditions to the workers' psychophysiological, which took place by putting together the operatory and the technical scientific knowledge. The ergonomic claim of the company being studied emerged from an internal need, and its main objectives were: (i) carry out an ergonomic analysis of the

\footnotetext{
${ }^{1}$ Footnote in title. Please ensure there is a 24 pt blank line before the title!

*Corresponding author. E-mail: contato@dfe-ergonomia.com.br.
} 
work post of the injection operators and carousel presses and of the workbench finishing, in order to diminish the ergonomic risks and to prevent LER/DORT, indicating the technical recommendations to reach this objective, observing the NR $16-$ MTE Ergonomics.

\section{Research Method}

The planning and the developing of the actions in order to meet this demand resulted from three stages: ergonomic analysis of the work post (stage $1-\mathrm{Jun} / 06$ to Dec/07), ergonomic improvements (stage 2 - from Jun/06 to current date) and the Ergonomic Committee (stage 3 - from Jun/08 to current date).

The method was based on the Ergonomic Analysis of Work proposed by Wisner (1994) and the stages of the ergonomic analysis are those presented in the 17 NR Regulatory Manual (MET, 2002), as indicated as follows. Ergonomic analysis methodology proposed by Wisner (1994, p. 96): (1) Contract proposal and demand analysis; (2) Technical, economic and social post analysis; (3) Tasks and work situation analysis and result feedback; (4) Ergonomic recommendations; (5) Intervention validations and recommendation efficiency.

Stages of the ergonomic analysis proposed in the 17 NR Regulatory Manual (MET, 2002, p. 17): (1) Context and demand analysis; (2) Company's global analysis; (3) Employee population analysis; (4) Work situation definition to be studied; (5) Description of the appointed tasks, of the real tasks, and of the developed activities to be executed; (6) Pre-diagnosis establishment; (7) Systematic observation of the activity as well as of the available resources to get the activity done; (8) Diagnose or diagnosis; (9) Diagnose validation; (10) Modification/alteration project; (11) Modification/alteration implementation schedule; (12) Modification/alteration follow up.

The methodological procedures applied were based on descriptive research techniques (interviews, verbalizations, questionnaires and observations), exploratory and qualitative criteria and guidelines available in literature and legislation, as well as company information (staffing, productivity, medical data, etc.).

This research has privileged the approach concerning the voluntary participation of the workers. The information gathered, however, was kept confidential.

The footages and the pictures were only made after getting the company's authorization and also after the workers who were participating have allowed as well.
The supporting materials used were: filed notebook, which consists of a notebook where data are gathered and kept, a video camera, a photographic camera and a data show.

\section{Case Study}

\subsection{The Work Ergonomic Analysis (stage 1)}

According to the demand, the ergonomic analysis focused on the work post of the injection operators (Plastic Sector) and of the PU carousel press operators and of the workbench finishing (Foaming Sector) of the production area observing the NR 17 - Ergonomics, specifically the 17.1.11 1nd 17.6.2 items.

In the Plastic Sector, the positive aspects highlighted by the workers were: good work post, freedom to do their jobs, benefits, good EPI's, good relationship with their workmates, continuous improvement in the sector. On the other hand, the negative points mentioned were: staff turnover, knives (insufficient available quantity), pieces that get stuck in the machinery, bigger work stations of the injector, the food that is served in the dining hall.

The main ergonomic embarrassments observed throughout the analysis were: 1) the inferior members overload associated with the work bodily position (they work standing); 2) dislocations (covered distances and frequency) associated with the handloading of pieces (unitary, small batches and boxes); 3) inappropriate conditions to those who have to work sitting down (improvised on KLTs); 4) torso inclination and torso flexion associated with the placing of pieces on racks and cabinets; 5) strength application associated with the technological restrictions of the equipment (for example: the use of a rubber hammer to attach a string in a medium injector piece; manually open and close the door of small injectors); 6) repetition of cycled movements inferior to $30 \mathrm{sec}-$ onds (in some pieces of small injectors).

In the Foaming Sector, the positive points mentioned by the workers were: knowledge/mastery of the job, being able to work sitting down, $3^{\text {rd }}$ shift implementation, exchange of the cell breaking engine. On the other way around, the negative points indicated were: the oiliness of the desmolder when in contact with parts of the body (the company, however, has been installing more fans) and insufficient seats.

The most severe embarrassments observed were: 1) overload of the inferior members associated with the work bodily position (they stand on their feet); 2) dislocations (covered distances and frequency) asso- 
ciated with the hand-loading of pieces; 3 ) torso bending, 4) extension and head suspension; 5) arm elevation and sustention (on the level of the shoulders); 6) light strength application associated with the withdraw of pieces of the molds; 7) intense work pace.

\subsection{Adjust of the work conditions (stage 2)}

In the Plastic Sector, it can be seen an readjustment of the work posts and the inclusion of physical resources (treadmills to move the pieces, shelves, higher seats, footboard) in order to help the workers to perform their activities when standing up or sitting down (posture alternation), when compared to the previous posture condition: walking or improvising a place where to sit on (like on KLTs), what would lead people to adopt painful postures.

As a long-term goal, the company has been investing in training courses for the workers (flexibility matrix) to expand the post rotation in order to reduce the exposition to repetitive activities, movement amplitude and weight handling, associated with the variations in the pieces' characteristic dimensions.

In the Foaming Sector, it can be identified a reorganization of the work and interventions in the rotation system of the carousel and finishing subsectors in order to reduce the exposition to the standing up and walking positions as well as the forced torso bending positions and the head ex-tension (imposed by the physical and dimensional characteristics of the carousel). It can also be seen an optimization of the carousel security system, an enlargement of work in the finishing posts and a readjustment of the workbenches (finishing subsector) concerning the performance of standing up and sitting activities (posture alternation), both previously accomplished in the standing up position.

\subsection{Ergonomics Committee (stage 3)}

The company's Ergonomics Committee (COERGO) was formed by voluntary workers from different sectors and different hierarchical levels. Among its attributions is the mission of giving continuity to the ergonomic process of the company by following the implementation of the improvements, the validation of the adjustments and the reevaluation of the work conditions.

\section{Final Considerations}

In June 2006, the company began the ergonomic analysis and since September of the same year it has been creating ergonomic improvements along with the effective participation of its workers from different areas and from different hierarchical levels. From the inconsistencies and recommendations pointed out in the ergonomic analysis of the adjustment of the work conditions to the worker' psychophysiological (stage 1) concerning the analyzed sector ergonomic improvements were made (stage 2) related to the handling of the materials, furniture, equipment, environmental conditions of the post work and to the work organization itself (17.1.1 - NR 17). In both sectors interventions were observed in the physical environment through improvements in the levels of comfort related to temperature (in the summer), lightening system (adjustment of the amount of light on the work area and the use of natural lightening) and the use of color.

After the whole process was finished, it could be observed: (i) the workers satisfaction (in relation participative approach towards the improvements implemented); (ii) the transference of expertise about ergonomics to the company, particularly the "knowhow" by asking and testing proposals involving the workers' participation; (iii) the appropriation of the ergonomic process by the company through the implanting of an Ergonomic Committee; and (iv) the inclusion of ergonomics in the organizational culture (by adding behavioral changes and attributing more importance to the ergonomic issues in different areas and decisions of the company). Regarding the external scope, the company is today a benchmark reference, concerning its ergonomic program, to other companies of its group and to other organizations which understand the importance of ergonomics to their employees.

Finally it is important to emphasize that these results are due to the set of efforts of the company involving different areas, specially, Human Resources, Engineering (process and security), Medicine and Law, and the sector workers under analysis.

\section{References}

[1] B. Newman and E.T. Liu, Perspective on BRCA1, Breast Disease 10 (1998), 3-10.

[2] D.F. Pilkey, Happy conservation laws, in: Neural Stresses, J. Frost, ed., Controlled Press, Georgia, 1995, pp. 332-391.

[3] B. Newman and E.T. Liu, Perspective on BRCA1, Breast Disease 10 (1998), 3-10. 
[4] D.F. Pilkey, Happy conservation laws, in: Neural Stresses, J.

Frost, ed., Controlled Press, Georgia, 1995, pp. 332-391.

[5] E. Wilson, Active vibration analysis of thin-walled beams,

Ph.D. Dissertation, University of Virginia, 1991.

E. Wilson, Active vibration analysis of thin-walled beams, Ph.D.

Dissertation, University of Virginia, 1991. 